\title{
A Simple Quantitative Assay for Bacterial Motility
}

\author{
By LEE A. SEGEL \\ Department of Applied Mathematics, The Weizmann Institute of Science, Rehovot, Israel* \\ and \\ Department of Mathematical Sciences, Rensselaer Polytechnic Institute, \\ Troy, New York, U.S.A. \\ ILAN CHET AND YIGAL HENIS \\ Department of Plant Pathology and Microbiology, Faculty of Agriculture, \\ Hebrew University of Jerusalem, Rehovot, Israel
}

(Received 20 January 1976; revised 7 September 1976)

SUMMAR Y

It is argued that the average motility of bacterial populations should be identified with a diffusivity parameter. A simple capillary assay for quantifying this parameter is described, and some results obtained by using this procedure are presented. It is concluded that the assay combines speed and simplicity of operation with sufficient accuracy to make it a valuable tool in the assessment of motility.

\section{INTRODUCTION}

The object of this communication is to argue that 'effective diffusivity' is the best single measure of bacterial motility, to describe a simple procedure for quantifying this measure, and to present some results obtained by using this procedure under various conditions.

Qualitative characterization of bacterial motility as 'good' or 'poor' is commonplace, but more precise and objective standards should now be employed. This communication is concerned with the quantitative characterization of the motility of a bacterial population. This is 'clonal motility' in the terminology of Iino \& Enomoto (I97I); perhaps 'population motility' is a better phrase as the population need not come from a single clone.

In the absence of specific stimuli, an individual bacterium moves about in a random fashion. This situation is comparable to the random motion of solute molecules in solution. In the latter case it is well established that the diffusion equation provides a description of how solute concentration changes, and that this description is in good accord with experiment for various sizes and shapes of solute and solvent molecules. Differing details of molecular motion affect the average concentration only through a single parameter, the diffusivity.

The observed randomness in the motion of solute molecules results from complicated interactions with (exterior) solvent molecules. In the case of bacteria, it is the complicated interactions of interior molecules that give rise to the appearance of randomness. But the fact, not the cause, of the randomness governs the phenomenology, and the details of the random motion are largely irrelevant to average behaviour. Thus to a first approximation,

\footnotetext{
* Address to which reprint requests should be directed.
} 
we expect changes of bacterial concentration to be governed by the classical diffusion equation (in the case of constant $\mu$ )

$$
\frac{\partial C}{\partial t}=\mu\left[\frac{\partial^{2} C}{\partial x^{2}}+\frac{\partial^{2} C}{\partial y^{2}}+\frac{\partial^{2} C}{\partial z^{2}}\right]
$$

in which $C$ is the concentration of bacteria (number per unit volume), $x, y$ and $z$ are spatial coordinates, $t$ is time, and $\mu$ is the motility coefficient (an effective diffusivity).

The general effect of diffusion is to level out differences in concentration. The larger $\mu$ is, the faster this process will take place, for $\mu$ measures the vigour of random motion. We urge that $\mu$ be adopted as a standard measure of population motility.

The numerical value of $\mu$ can be determined in many ways (these are, of course, analogous to the ways in which solute diffusivity is quantified, or heat diffusivity is measured). All methods involve comparison of the observed changes in an initial distribution with a theoretical solution of the diffusion equation (1). For example, one could observe the spread of bacteria initially confined to a small drop, or the change in concentration after a tube is initially half-filled with a bacterial suspension. The corresponding theoretical concentration distributions are recorded in books such as that of Crank (I956).

\section{METHODS}

We investigated the following method of determining $\mu$. (a) A capillary tube of crosssectional area $A$ is filled with medium. (b) The open end of the tube is placed in a suspension of bacteria of known concentration $C_{0}$. (c) The tube is removed after time $T$ and the number $(N)$ of bacteria that it contains is counted. (This time should be selected so that appreciable numbers of bacteria are found at a distance of several tube radii from the open end. The tube should be long enough to ensure that few bacteria reach its closed end.) $(d) \mu$ is computed according to the formula

$$
\mu=\pi N^{2} / 4 C_{0}^{2} A^{2} T
$$

The derivation of formula (2) is discussed in the Appendix.

Experiments were performed to check that formula (2) yielded consistent results under a variety of conditions. The test organism Pseudomonas fluorescens was grown on nutrient agar (Difco) for $24 \mathrm{~h}$. The bacteria were washed from the plates with O.I M-phosphate buffer $\mathrm{pH} 7 \cdot 0$. All cultures were transferred to buffer 5 to $\mathrm{I} 2 \mathrm{~h}$ before use. Response was determined using the capillary methods of Adler (1969). Capillaries with a capacity of I $\mu$ l (length $32 \mathrm{~mm}$ ) were filled with phosphate buffer and closed at one end. The open end was placed in a bacterial suspension on a glass slide. The number of bacteria that entered the capillary after the prescribed time was determined by diluting its contents with buffer and plating portions on nutrient agar. Colonies were counted after 3 days, and their number was multiplied by the dilution factor. In the first experiments, the capillaries were closed with a silicone grease; later an agar plug was used, which was easily removed with the bacteria into the buffer. This modification reduced the standard error.

Errors can easily occur because of the many dilutions, pipetting operations and transfers involved in this simple counting method. In our experiments the average standard errors calculated from the replicates was 15 to $18 \%$. Experience indicated that five or six replicates provided a relatively low standard error without undue labour. 
Table I. The effect of the duration $(T)$ of the test on the number $(N)$ of bacteria

Bacterial concentration in the suspension $\left(C_{0}\right)$ was $7 \times 10^{7} \mathrm{ml}^{-1}$.

$\begin{array}{cc}\text { Time } T(\mathrm{~min}) & N \\ 2 & 1800 \\ 5 & 3700 \\ 10 & 4800 \\ 12.5 & 5500 \\ 15 & 6700 \\ 20 & 8000^{*}\end{array}$

* One very high count was rejected, so only four values were averaged.

Table 2. The effect of the bacterial concentration in the suspension $\left(C_{0}\right)$ on the number $(N)$ of bacteria in the capillary after $10 \mathrm{~min}$

$\begin{array}{cc}\mathrm{IO}^{-7} \times \text { Concn }\left(C_{0}\right) & \\ (\text { bacteria ml } & -1) \\ 2 \cdot 5 & N \\ 4 \cdot 6 & 1350 \\ 5 \cdot 0 & 2300 \\ 12 \cdot 0 & 3400 \\ & 6200\end{array}$

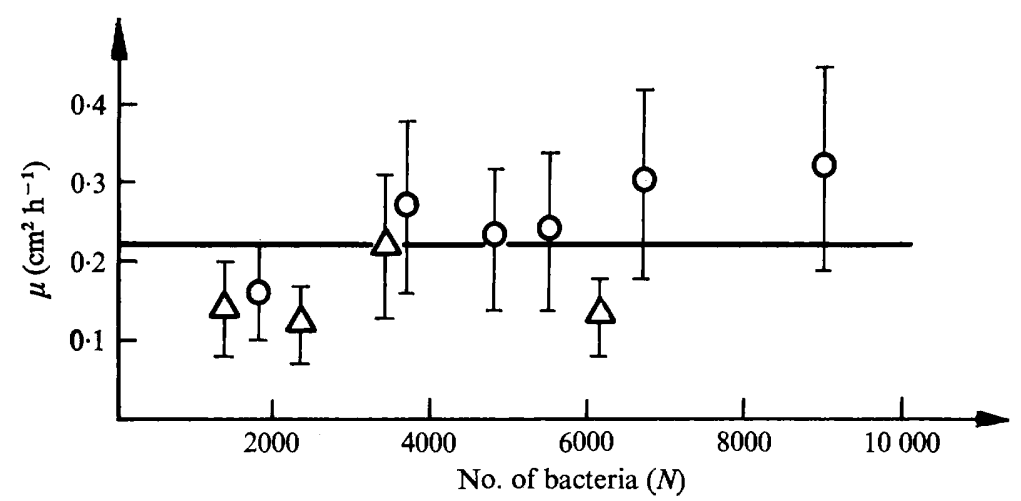

Fig. I. Values of motility coefficient (effective diffusivity) calculated on the basis of equation (2). Circles show data of Table I, triangles show data of Table 2, for the number $(N)$ of bacteria found in the capillary. Standard errors are indicated.

\section{RESULTS}

The effects of the duration $(T)$ of the test and the concentration $\left(C_{0}\right)$ of the bacterial suspension on the number $(N)$ of bacteria that entered the capillary were measured. The results are shown in Tables $I$ and 2 : each result is the average of five experiments.

The data from Tables $I$ and 2 are plotted in Fig. $I$. The numbers of bacteria in the tubes varied considerably, owing to the different experimental conditions. Nonetheless, an average bacterial motility of about $0 \cdot 2 \mathrm{~cm}^{2} \mathrm{~h}^{-1}$ clearly gives the main trend of the results. A standard error of less than $20 \%$ in the bacterial count leads to a standard error of about $40 \%$ in the determination of $\mu$. This error has been indicated on Fig. I.

To see the effect of ambient conditions on motility, ethanol $(0.2 \%, v / v)$ and nutrient broth 
$\left(0.8 \mathrm{mg} \mathrm{ml}^{-1}\right)$ were added separately to the bacterial suspension (but not to the capillary). With ethanol, after Io min 5800 bacteria were found in the capillary; with nutrient broth, 32000 bacteria were found. The control experiment, in which only phosphate buffer was present, yielded a count of 7000 bacteria. It appears that ethanol somewhat depresses motility, particularly when possible negative chemotaxis is taken into account. Moreover, even though a degree of positive chemotaxis would tend to drive bacteria out of the capillary, the results show at least a 20 -fold increase in motility in nutrient broth. We emphasize that these results concern random motility, not chemotaxis.

\section{DISCUSSION}

\section{Chemotaxis and motility}

The determination of bacterial motility has acquired added interest of late in view of current attempts to examine the effects of various chemicals on bacterial movement. The modern revival of interest in this subject was initiated by J. Adler and his associates. One of their earliest papers (Adler \& Dahl, 1967) defined a 'motility coefficient' as a diffusivity like $\mu$, and attempted to measure it by comparing the spread of an initial discontinuity with diffusion theory. A possible difficulty with these experiments is that the bacteria exhibit chemotaxis in gradients of the substances used in the medium, and they metabolize those substances so gradients must be created. Adler \& Dahl (1967) omitted a chemical that was necessary for the formation of travelling bands of chemotactic bacteria, but the absence of bands does not necessarily imply the absence of chemotaxis.

Perhaps a more important possible source of inaccuracy arose from the fact that Adler \& Dahl (1967) based their assay on the course of the 'leading edge' of a diffusing bacterial population and thus estimated the motility of the fastest-moving bacteria. Whatever the source of disagreement, Adler \& Dahl (1967) reported results that were considerably at variance with diffusion theory. These authors were nonetheless the first to propose the use of the effective diffusivity as a parameter of importance, and their results certainly provide an estimate of this parameter.

Adler \& Templeton (1967) followed the extent of 'diffusion' of bacteria inoculated in one end of a capillary to study the effect of environmental conditions on the motility of E. coli. Later Adler (1973) suggested the use of a capillary assay for motility and provided important advice for its successful use. The major contributions of the present communication are (i) its stress on the fact that $\mu$ provides an objective measure of motility which is not bound to any particular experimental procedure and (ii) its presentation of formula (2) for quantifying $\mu$ in the various capillary assays for motility.

Adler (1969) used a capillary assay to measure the strength of chemotaxis: his assay was essentially the same as that recommended here for the assessment of motility. (Adler points out that the qualitative procedure was used by W. Pfeffer in the I880s.) However, it is difficult to relate the number of bacteria found in the tube with a clearly defined measure of chemotaxis because the rate at which the bacteria enter the tube depends on the gradients in chemical concentration and also on the motility. Futrelle \& Berg (I972) have taken a first step by providing an approximate solution to the problem of chemical diffusing from a capillary into a reservoir.

The role of motility in situations where chemotaxis occurs is clarified by considering what happens to a bacterial population faced by a possibly non-uniform concentration of chemical attractant. Considering, for simplicity, a one-dimensional situation for which $b(x, t)$ denotes the bacterial concentration at position $x$ and time $t$, and $s(x, t)$ denotes the chemical con- 


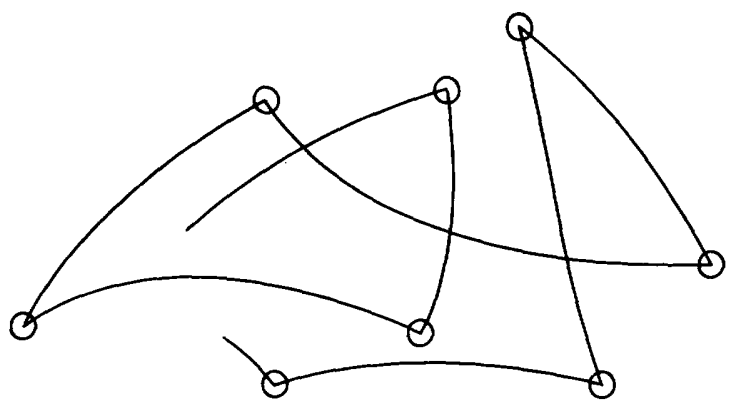

Fig. 2. A possible trajectory of a bacterium. The circles mark turning regions. The 'progress' of a bacterium and the spread of a bacterial population depends not only on how fast the bacteria swim, but also on how frequently they turn.

centration, then it has been argued (Keller \& Segel, 197I $a$; Segel, 1976) that the evolution in time of $b$ is governed by the equation

$$
\frac{\partial b}{\partial t}=-\frac{\partial}{\partial x}\left[-\mu(s) \frac{\partial b}{\partial x}+\chi(s) b \frac{\partial s}{\partial x}\right]
$$

Here $\mu$ is the motility (already defined) and $\chi$ is a chemotactic sensitivity. The latter is the proportionality factor between net flow rate per unit bacterial concentration and substrate gradient. The drift velocity with which bacterial populations move up a gradient is given by $\chi \partial s / \partial x$.

Comparison between theory and experiment has shown that equation (3) can provide a satisfactory description of the chemotactic motion of bacterial populations (Keller \& Segel, 1971 $b$; Segel \& Jackson, 1973). Details of this comparison are not relevant here but three points should be stressed. (I) It is precisely $\mu$, and no other measure of random motility, that influences the motion of bacterial populations. (2) Possible dependence of $\mu$ on the substrate level $s$ must be ascertained before the true strength of the chemotactic response $\chi$ can be estimated from any experiment. (3) Knowledge of $\mu(s)$ and $\chi(s)$ is of considerable value in an investigation of chemotaxis. Plausible models of the behaviour of an individual bacterium can immediately be ruled out, for example, if appropriate averages do not yield the observed dependences of $\mu$ and $\chi$ on the attractant concentration $s$ (Segel, 1976, 1977).

\section{Relation of the motility coefficient to other parameters}

A measure of bacterial speed is sometimes used to quantify 'motility'. To examine the relation between such a speed and $\mu$, we first note that the trajectory of a typical bacterium might resemble the line in Fig. 2. In principle, an average speed $v$ can be determined from observations of a number of bacteria over relatively straight portions of their trajectories. But another parameter is required to characterize the motion, the mean free path $\lambda$, i.e. the average distance between turns. It can be shown (Lovely \& Dahlquist, 1975) that if turns provide a totally random new direction then the diffusivity $\mu$ is related to $v$ and $\lambda$ by the formula

$$
\mu=\frac{1}{3} \nu \lambda
$$

Thus, although both the mean free path $\lambda$ and the average speed are often of interest for 
specific purposes, it is only the product of these quantities, as they appear in formula (4) for the motility coefficient $\mu$, that influences the motion of a population of bacteria.

Knowing the dependence of motility on speed, one can gauge the effect of the viscosity of the medium in which the cells are suspended. The speed $v$ of a cell is proportional to the force $F$ that is driving it through a fluid of viscosity $\eta$ according to the formula

$$
F=k \eta v
$$

Here $k$ is a geometry-dependent friction coefficient that can in principle be determined by calculations of 'low Reynolds number fluid mechanics'; for a sphere of radius $a, k=6 \pi a$ (Stokes drag law). Combining equations (4) and (5), we see that if cells travel essentially the same mean free path $\lambda$, regardless of the medium in which they are suspended, then

$$
\mu=\left[\frac{F \lambda}{3 k}\right] \frac{\mathrm{I}}{\eta}
$$

However, equation (4) can be rewritten in terms of the mean time between turns $\tau$, where $\tau=\lambda / v$ :

$$
\mu=\frac{1}{3} \tau v^{2}
$$

If $\tau$ is essentially independent of viscosity (which seems likely for bacteria like $E$. coli or Salmonella that perform distinct tumbles) then

$$
\mu=\left[\frac{\tau F^{2}}{3 \bar{k}^{2}}\right] \frac{\mathrm{I}}{\eta^{2}}
$$

Note that in equation (6) the motility is inversely proportional to the fluid viscosity $\eta$, whereas in equation (8) the proportionality is to the inverse square of the viscosity. [The numerical factor $\frac{1}{3}$ in these formulae should be regarded as approximate for the factor will be different if there is any correlation between the bacterium's directions of movement before and after its turns (see, for example, Lovely \& Dahlquist, 1975).]

The discussion of the previous paragraph was concerned solely with the fluid mechanical effect of viscosity changes on the motility $\mu$, assuming no change in the swimming mechanism. By contrast, Shoesmith (1960), Berg \& Brown (1974) and Schneider \& Doetsch (1974) have demonstrated that in many circumstances bacteria swim faster at higher viscosities. Attempts to understand this surprising behaviour must begin with a knowledge of how it differs from the motion predicted from a strictly mechanical argument.

\section{Errors}

We have mentioned chemotaxis as one source of error in our motility assay. Another is the depletion of oxygen in the tube; this may lead to a cessation of motility or to a change to an anaerobic mode of operation. Accumulation of waste products may also lead to motility changes. These three sources of error will be minimized if the bacterial population is kept as low as is otherwise feasible. Errors that can be introduced by the use of equation (2) are discussed in the Appendix, and counting errors have been mentioned above. Adler (r973) stresses the importance of clean capillaries and beakers.

Adler (I973) found about Ioo non-motile bacteria in the tube after I h. About 30 would be expected on the basis of Berg \& Brown's (1975) rough calculation of the expected diffusivity of such an organism $\left(\mu \approx 0.3 \times 10^{-4} \mathrm{~cm}^{2} \mathrm{~h}^{-1}\right)$ 'computed from Stokes law for a $2 \mu \mathrm{m}$ diameter sphere in a medium of viscosity $2.7 \mathrm{cP}$ at $32{ }^{\circ} \mathrm{C}$ '. Like Adler, but to a greater degree, we found more non-motile bacteria in the capillary than expected on the basis of Brownian 
motion. [After 5 (10) min, starting with an initial concentration of $5 \times 10^{7}$ cells ml $^{-1}, 270$ (400) micrococci entered the capillary.] It appears that there is a small systematic error of some kind; it could be a form of convection, although experiments were run for a comparatively short time, in the dark and at constant temperature, in order to minimize this difficulty.

One more source of error is mentioned in the concluding paragraph.

\section{Other possible assays}

More accurate determinations of $\mu$ can be made if desired, but these require more than a simple capillary. For example, quite precise values of $\mu$ can be obtained using the apparatus of Dahlquist, Lovely \& Koshland (1972) which enables observation of the evolution of an initial distribution of bacteria (F. W. Dahlquist \& P. S. Lovely, unpublished). Indeed, using equation (3), values of $\mu$ can be calculated from existing data concerning the accumulation of chemotactic bacteria at a 'knee' in attractant concentration (Segel \& Jackson, 1973). Tracking devices that follow the movement of a single bacterium (Berg \& Brown, 1972; Lovely et al., 1974) can also be employed to determine $\mu$. Results of this type are given by Lovely \& Dahlquist (1975). Another new approach characterizes bacterial motion by the use of laser light scattering techniques (see review by Boon, 1975).

Nossal \& Chang (1976) determined $\mu$ for cells moving on a surface by analysing fluctuations in the number of cells occupying a defined area; this idea could also be used in threedimensional motion.

\section{Some results of the capillary assay}

Our experiments yielded a value of about $0.2 \mathrm{~cm}^{2} \mathrm{~h}^{-1}$ for the motility of starved Pseudomonas fluorescens. This is slightly lower than the value $0.25 \mathrm{~cm}^{2} \mathrm{~h}^{-1}$ obtained by Adler \& Dahl (1967) for the 'maximum motility' of E. coli $\mathrm{K} 12$ in the presence of an energy source, despite the fact that the latter organism is readily recognized to be 'less motile' than the former. Reasons for our belief that the Adler \& Dahl value is an overestimate have been given above.

Adler (1969) reported that a 'background value' of 3000 was subtracted from the counts used in his chemotaxis assay $\left(C_{0}=5 \times 10^{7}\right.$ bacteria $\left.\mathrm{ml}^{-1}\right)$ since this was the number of bacteria which entered the tube in $I h$ in the absence of attractant. From these data we can determine a value for $\mu$ of $0.03 \mathrm{~cm}^{2} \mathrm{~h}^{-1}$ for the bacteria Adler studied, i.e. E. coli $\mathrm{K}$ I 2 utilizing an endogenous energy source. In later experiments on a different strain of $E$. coli, Adler (I973) reported that I 1000 bacteria entered the tube after $90 \mathrm{~min}$ from a suspension whose concentration was nearly the same as that in the earlier experiments. This result provides an opportunity to demonstrate the usefulness of having a characterization of motility that is not bound to a particular experimental procedure. The second of the two E. coli motility counts is for a period $\mathrm{I} \cdot 5$ times as long as that for the first count. This difference is almost certainly significant, for according to equation (2), a factor of $\mathrm{I} \cdot 5$ in assay time $T$ should only be responsible for a factor of $\mathrm{I} \cdot 5^{\frac{1}{2}} \approx \mathrm{I} \cdot 2$ in count. Thus, the motility of the organisms used in the second set of experiments appears to be nine times the motility of those used in the first experiments.

\section{Final remarks}

It seems reasonable to conclude that 'effective diffusivity' is probably the best single quantitative characterization of average bacterial motility, yet a difficulty remains. As pointed out by Segel \& Jackson (1973) and Thonemann \& Evans (1976), our discussion applies to a uniform population of organisms with identical average behaviour. Actually 
each bacterium can be regarded as characterized by an individual value of $\mu$. The average of these values is accurately obtained by diffusion theory only if the distribution of individual values is tightly clustered about the mean. We have already mentioned that Adler \& Dahl's motility measurements were probably affected by a significant spread in motility values throughout the population (this matter is discussed in detail by Thonemann \& Evans, 1976). A similar effect seems to be discernible in our experiments. As can be seen from Fig. I, and is confirmed by a statistical analysis, it is likely that there is an increase in computed motility for later times in the trials listed in Table I. This may occur because, as time goes on, the bacterial population in the tube becomes proportionately enriched by fastermoving bacteria. This 'distribution' problem makes it difficult to provide a precise value for average population motility, for computation of the average requires knowledge of the size of subpopulations that are 'extremely motile', 'very motile', etc. However, as we have illustrated, much can be learned from a quantitative characterization of motility even if it cannot be regarded as precise. Because of this, and in spite of the errors which we have pointed out, we feel that the capillary assay combines speed and simplicity of operation with sufficient accuracy to make it a valuable tool in the assessment of motility.

\section{APPENDIX}

\section{Derivation of equation (2) for the motility coefficient $\mu$}

The assumptions used in deriving equation (2) were: (i) bacterial motion can be described by the diffusion equation (I); (ii) the tube is infinitely long; (iii) no bacteria pass through the wall of the tube, but bacterial motion is otherwise unaffected by proximity to the wall; (iv) from the first moment of the experiment the bacterial concentration at the open end of the tube is $C_{0}$. Given these assumptions, equation (2) follows from a simple known solution to the diffusion equation (I) (Crank, I956, p. I9). Assumptions (i) and (iii) should not be the cause of appreciable error. In particular, growth should be negligible considering that the bacteria were starved and the duration of the experiment was fairly short compared with the generation time. Since the experiment is terminated before more than a few bacteria have had a chance to reach the end of the tube, the bacterial population effectively does not 'realize' that the tube is finite in length. Thus negligible error should result from assumption (ii).

Assumption (iv) is definitely violated for short times ( $T$ ). Indeed, at the very start of the experiment the bacterial concentration at the open end of the tube immediately drops to $C_{0} / 2$, the average of its value inside and outside the tube. Only after a period of time does it build up to $C_{0}$ again, so equation (2) provides an underestimate of $\mu$. We expect that this build-up occurs in the time it takes the bacteria to 'diffuse' a distance of a few tube radii. General principles of diffusion theory [confirmed by the analysis of Futrelle \& Berg (1972)] show that this time will be perhaps $10 \times a^{2} / \mu$, where $a$ is the tube radius. For the capillary normally used, $a$ is $0.0 \mathrm{I} \mathrm{cm}$, and a typical value of $\mu$ is $0 . \mathrm{I} \mathrm{cm}^{2} \mathrm{~h}^{-1}$. Using these values we estimate the time taken for the concentration at the open end of the tube to be close to $C_{0}$ is $10^{-2} \mathrm{~h}$, i.e. about half a minute. In a $\mathrm{IO}$ min assay the error in using equation (2) should therefore be small.

The work of one of us (L.A.S.) was partially supported by a National Science Foundation Grant to Rensselaer Polytechnic Institute. 


\section{REFERENCES}

ADLER, J. (1969). Chemoreceptors in bacteria. Science 166, $1588-1597$.

ADLER, J. (1973). A method for measuring chemotaxis and use of the method to determine optimum conditions for chemotaxis by Escherichia coli. Journal of General Microbiology 74, 77-9I.

AdLeR, J. \& DAHL, M. (1967). A method for measuring the motility of bacteria and for comparing random and non-random motility. Journal of General Microbiology 46, I6I-1 73.

AdleR, J. \& Templeton, B. (1967). The effect of environmental conditions on the motility of Escherichia coli. Journal of General Microbiology 46, I75-I 84.

Berg, H. C. \& Brown, D. A. (1972). Chemotaxis in Escherichia coli analysed by three-dimensional tracking. Nature, London 239, 500-504.

Berg, H. C. \& Brown, D. A. (1974). Addendum to a reprint of the previous reference. In Chemotaxis, Biology and Biochemistry, Antibiotica et Chemotherapia, vol. 19, pp. 66-77. Edited by E. Sorkin. Basel: S. Karger.

Boon, J. P. (1975). Theoretical models for bacterial motion and chemotaxis. Advances in Chemical Physics 29, $169-190$.

Crank, J. (1956). The Mathematics of Diffusion. Oxford: Clarendon Press.

DAHLQUIST, F., LOVELY, P. \& Koshland, D. (1972). Quantitative analysis of bacterial migration in chemotaxis. Nature, New Biology 236, $120-123$.

Futrelle, R. P. \& BeRG, H. C. (I972). Specification of gradients used for studies of chemotaxis. Nature, London 239, $517-518$.

IINo, T. \& ENOMOTo, M. (197I). Motility. Methods in Microbiology 5A, I45-163.

Keller, E. F. \& SEGEL, L. A. (I97I a). Model for chemotaxis. Journal of Theoretical Biology 30, 225-234.

KeLLER, E. F. \& SEGEL, L. A. (I97I $b$ ). Travelling bands of chemotactic bacteria: a theoretical analysis. Journal of Theoretical Biology 30, 235-248.

Lovely, P., Dahlquist, F. W., Macnab, R. \& Koshland, D. E., JR (1974). An instrument for recording the motions of microorganisms in chemical gradients. Review of Scientific Instruments 45, 683-686.

Lovely, P. \& DAHLQUIST, F. (1975). Statistical measures of bacterial motility and chemotaxis. Journal of Theoretical Biology 5o, 477-496.

Nossal, R. \& ChANG, Y. T. (1976). A procedure for determining mobility parameters for cells moving along surfaces. Journal of Mechanochemistry and Cell Motility 3, (in the Press).

SCHNEIDER, W. R. \& DOETSCH, R. N. (1974). Effect of viscosity on bacterial motility. Journal of Bacteriology II7, 696-701.

SEGEL, L. A. (1976). Incorporation of receptor kinetics into a model for bacterial chemotaxis. Journal of Theoretical Biology 57, 23-42.

SEGEL, L. A. (1977). A theoretical study of receptor mechanisms in bacterial chemotaxis. SIAM Journal of Applied Mathematics (in the Press).

SEGEL, L. A. \& JACKSON, J. L. (1973). Theoretical analysis of chemotactic movement in bacteria. Journal of Mechanochemistry and Cell Motility 2, 25-34.

SHOESMITH, J. G. (1960). The measurement of bacterial motility. Journal of General Microbiology 22, 528-535.

ThonemanN, P. C. \& Evans, C. J. (1976). The dispersal of an initial concentration of motile bacteria. Journal of General Microbiology 92, 25-3I. 